\title{
Views of University Administrators on the Outsourcing of Online Education
}

\author{
Evan Silberman \\ New York University
}

This paper details the findings of a case study conducted at a 4-year private university in the United States that outsourced online learning through university-industry partnerships. The case study was conducted to reveal university administrators' views on (a) working with online program management providers (OPMs) to design, deliver, and implement online learning and (b) how online learning became an institutional practice. The study findings indicated that the university's culture of innovation resulting from institutional support in the form of governances, grants, and services led to university administrators' decision to outsource. The study findings also indicated that university administrators did not trust OPMs because the OPMs did not meet university administrators' expectations. University administrators expected OPM staff to (a) have advanced degrees and experience working in higher education, (b) understand the academic discipline for the program they were supporting, and (c) understand higher education practices and policies. Lastly, the study findings revealed that decision-making is an essential process to promote trust and reduce conflicting expectations.

Keywords: outsourcing, online program management providers, OPMs, university-industry partnerships, higher education, online learning, teaching and learning, $O P X$

\section{INTRODUCTION}

School closures resulting from COVID-19 disrupted nearly 1.2 billion students and youth worldwide (Giannini, 2020). In the United States, 14,000,000 students across 4,000 postsecondary institutions experienced an unprecedented shift to online learning (Entangled Solutions, 2020). Prior to the global pandemic, on-campus enrollment in the U.S. higher education system was declining while enrollment in online courses and programs was on the rise (Seamen et al., 2018). These trends underscore the importance of online learning to higher education and highlight the need for postsecondary institutions to rapidly and sustainably bring programs online.

One-way universities accomplish online learning is through partnerships with online program management providers (OPMs). OPMs are for-profit companies that work with higher education institutions to build, recruit for, and deliver online programs for either a revenue share (sometimes up to $80 \%$ ) or a contracted fee-for-service model (Hill, 2019). The OPM market is emerging. OPMs and similar providers accounted for nearly 1,000 university partnerships over the past 2 decades (Holon IQ, 2020). Sixty OPMs currently exist in a $\$ 3,000,000,000$-dollar marketplace (Holon IQ, 2019). Some forecast that $50 \%$ of higher education institutions will be outsourcing essential support functions for their core mission of teaching and learning by 2024 (Morgan, 2019). 
OPMs fall into three categories: general-purpose, platform-based, and boot-camp OPMs. Generalpurpose OPMs offer bundled and unbundled services across the student life cycle; they are considered the most competitive segment and work mainly with graduate degree programs. Platform-based OPMs emphasize recruitment for their partner institutions and, as such, leverage their "platform as a presence" (Morgan, 2019, p. 5). Lastly, boot-camp OPMs work with institutions to deliver noncredit learning pathways (Morgan, 2019). Across these segments, OPMs provide turnkey services such as recruitment and enrollment, faculty and student support, technology services and platforms, course design and development, and student placement in internships and practicums (Morgan, 2019). University-industry partnerships with OPMs are essential for universities that do not have the resources to plan, deliver, and implement online programs. University administrators are at the center of these university-industry partnerships and administrators are responsible for the partnerships and the techniques, practices, protocols, and processes related to them (Martin, 2000; Slaughter \& Leslie, 1997).

\section{RESEARCH STUDY}

This paper discusses a qualitative case study implemented at Big U (pseudonym), a private, 4-year research university in the United States with an expansive global footprint. Big U had approximately 25 online graduate degree programs, eight of which were developed through a partnership with an OPM. Four OPMs were contracted to work with staff in three different graduate schools to create and deliver six online masters and two online doctorate programs. I choose Big U for this study because of its partnership with multiple OPMs. Throughout this case study, I examined university administrators' views on the outsourcing of online learning at Big $\mathrm{U}$ and how online learning became an institutional practice at Big $\mathrm{U}$.

\section{Data Collection}

I interviewed 34 individuals for this study: two associate professors, two chief information officers, one chief of staff, one chief operations officer, five deans, five directors, three program coordinators, six program directors, two project managers, two vice provosts, one vice president, and two external consultants. All participants were either involved in online learning or working with OPMs to plan, design, or deliver online programs. I promised participants that their identity and the name of their institution or organization would be kept anonymous.

I grouped interview questions into two broad categories to address this study's different goals: (a) perceptions of working with OPMs and (b) the history of online learning. Only select participantsparticularly the two vice provosts and a vice president—had the historical knowledge to discuss how online learning became an institutional practice; therefore, these participants were only asked about the history of online learning, whereas I asked all other participants about their beliefs and perceptions of working with OPMs. I conducted interviews in-person and recorded all of them. Interviews were 60-90 minutes in duration.

Additionally, other documents were collected and analyzed to triangulate interview data and provide additional context for this case. Documents were identified from public websites or shared by participants. Documents included strategic plans, policies, procedures, memos, reports, meeting records, and websites.

\section{Data Analysis}

Qualitative research can be conducted in many ways; however, most qualitative research shares a standard set of attributes (Miles \& Huberman, 1994). This study leveraged established data analysis practices, such as those articulated by Miles et al. (2019): data collection, data display, data condensation, and drawing and verify conclusions. This study also included triangulation, peer review, thick descriptions, and member checking.

I started the data analysis process (see Figure 1) for this study early in data collection to help me think about the data in new ways and improve data collection iteratively to avoid blind spots, energize the process, and substantiate formative reports that informed later parts of the analysis (Miles \& Huberman, 1994). Early 
data analysis in this study included field notes during the interview, marginal remarks on transcripts to inform coding, and analytical memos to conceptualize pieces of data (Miles \& Huberman, 1994).

I coded the data using Saldaña's (2016) two-cycle coding process. In the first cycle, I used a deductive approach and developed "a priori codes" (Saldaña, 2016, p. 175) based on the literature review, research questions, marginal remarks, and prior knowledge of the field. I developed a set of 48 codes and categorized them according to 11 main areas. These areas were (a) background, (b) decision making, (c) views of OPMs, (d) definitions of partnership, (e) success, (f) views of online learning, (g) institutional support, (h) the pursuit of online learning, (i) values, (j) governance models, and (k) uncertainty about online learning. Next, I coded the transcripts, starting with this a priori set of codes. Through an iterative process, I entered the next cycle of coding with 80 codes.

In the second cycle, I used "pattern coding" to identify emerging "categories, themes, and concepts" (Saldaña, 2016, p. 236). I analyzed frequencies of individual codes to determine which codes had the most occurrences. Then, I categorized the codes into two broad categories-a background of the case and assessments of OPMs - which allowed me to see commonalities across the codes. Within these two categories, I consolidated codes that represented similar phenomena and eliminated or dropped codes unrelated to the research questions. Next, I developed assertions and explored them based on the codes from each category. The assertions were (a) university administrators do not trust OPMs, (b) university administrators do not view the OPM relationship as a partnership, which reflects unmet expectations of the OPM, (c) institutional support is essential for online learning and the outsourcing of it, and (d) decisionmaking and decision implementation processes are critical to a successful OPM partnership.

These assertions led to two themes: (a) organizational change and innovation and (b) missed expectations and trust. Within each theme were a set of related subthemes.

\section{FIGURE 1 \\ DATA ANALYSIS PROCESS}
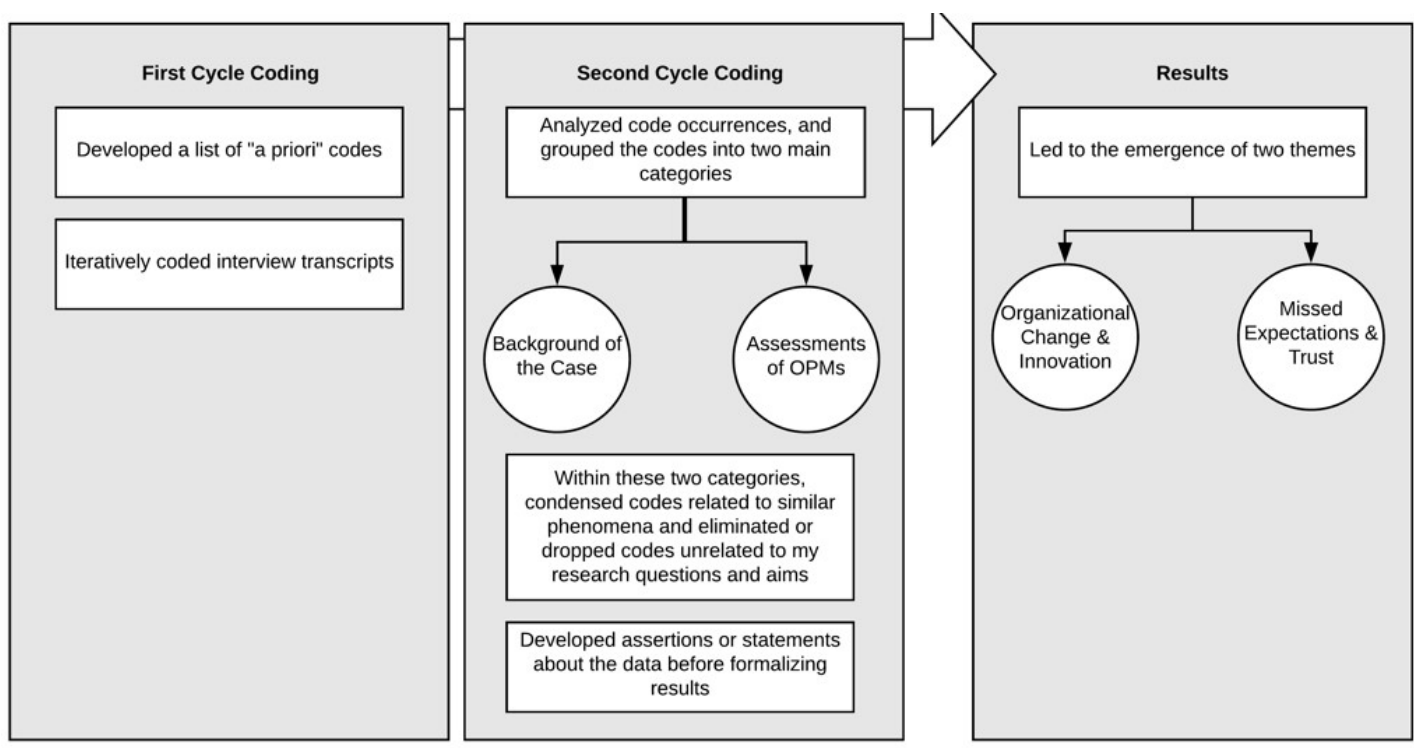

The results that follow reflect the data analysis process described above. The results explain the two themes_-organizational change and innovation and missed expectations and trust—and a vital subtheme: decision-making. I begin with decision-making because it has a direct practical implication for institutions considering OPM partnerships. 


\section{RESULTS}

Thirty-four interviews were conducted and data were grouped to develop a set of recommendations for practitioners in higher education. The following are a set of themes that emerged from the data collected and analyzed for this study.

\section{Decision-Making}

The decision-making process is essential to a successful OPM partnership. Decision-making can promote mission alignment and reduce miscommunication, conflicting expectations, and misunderstandings that lead to problematic partnerships (Ren et al., 2009).

Decision-making must involve expert administrators and tends to commit institutions to ventures that have both a direct and indirect impact on academic programs and issues. For instance, starting an online program may divert resources away from current in-person programs. An associate director believed this was the OPMs influence, explaining that "they [the OPM] want this institution to preference online students" (Participant 18, personal communication, October 4, 2019). A program director was more specific: "There is a pool of money available for online students that's not available for on-campus students. And that's an effort to build the online program" (Participant 3, personal communication, November 5, 2019). Administrators believed that online programs and students were favored over on-campus programs and students.

This inequity of resources may partially be the result of the administrators' absence in the decisionmaking process. The OPMs at Big U were often vetted and contracts were executed by senior leaders (e.g., school deans, vice presidents, and general counsel). In some cases, faculty members and administrators were invited to partner presentations; however, administrators at Big U revealed that the decision for their program to work with an OPM was not necessarily democratic. A program director shared that "the administration has decided that this new program is online" (Participant 8 , personal communication, October 22, 2019). This program director shared that she was in a faculty meeting when she learned her program was selected to be built online in partnership with an OPM. In recounting the story, this program director explained, "I left that meeting and realized there were discussions that have been taking place between the administration and [the OPM], and the faculty had been paying no attention to any of it" (Participant 8, personal communication, October 22, 2019). Institutions will benefit from involving an administrator in the decision to hire OPMs.

A program coordinator explained, "you must have people who actually do the grunt work at these meetings. So, not necessarily the deans and directors. People who are working with students" (Participant 10 , personal communication, October 3,2019). University administrators in this study believed that their involvement in the decision-making process would have benefited the institution and the partnership because of their experience operationalizing academic programs.

\section{Missed Expectations and Trust}

In the absence of an inclusive decision-making process, university administrators involved in the dayto-day work with OPMs established their own expectations of the OPMs. These expectations were based on administrators' (a) previous experience with online education, (b) exposure to OPMs, including personal experience or stories heard from the media and peers, (c) academic role and level of involvement with the partnership, (d) experience working in higher education, (e) experience with the traditional graduate education, (f) personal and professional goals, and (g) beliefs about higher education and for-profit businesses. Mismatched expectations contributed to the negative perceptions of trust that university administrators expressed towards OPMs.

Administrators' expected OPM staff to have experience working in education and to hold advanced degrees. Administrators viewed OPM staff favorably when the staff met these expectations. A departmental administrator explained, "I think some of their experts were helpful. There were folks who were PhDs, and professors, and retired professors who were really interested in this new innovative way" (Participant 13, personal communication, September 27, 2019). A program director compared two OPM staff members who 
held a similar role as general manager at different times during the partnership. The OPM general manager who held a Ph.D. was viewed positively. The program director explained, "He has a Ph.D. and has had roles in working with charter schools. I think we were all confident he truly does believe in education" (Participant 15, October 9, 2019). However, the partnership was viewed as problematic when this program director was working with an OPM general manager without the right background: "His interaction style and [he] doesn't have the academic background ... the trust I don't think is there" (Participant 15, October 9, 2019).

Administrators' also expected OPM staff to understand the academic discipline of the program they were supporting. Several of the OPMs provided field placement services where the OPM aided the program by placing students in internships and practicums for credit. An assistant program director explained the role of the OPM staff:

What really struck me was that the people that were working with us were not [physical therapists]. I'm working with individuals that are going to be helping to place our students, but I would have to be the ones training them in the requirements, in our accrediting body, in the culture of [physical therapy] and what the expectations are on the outside. (Participant 9, personal communication, November 4, 2019)

This conflicting expectation created distrust between administrators and OPM staff due to the OPM staffs' lack of knowledge about the program's academic discipline.

Lastly, administrators expected the OPM staff to understand how higher education worked. For example, one of the participants shared a story about an OPM staff member who unknowingly violated the Family Educational and Privacy Rights Act (FERPA), a federal law the protects the privacy of student data. The OPM staff pulled student information, including grades, and circulated the information in an e-mail with people who were not FERPA eligible. The associate director discussed this issue: "I think as university business partners, I trust them to understand what they do and the importance of FERPA" (Participant 18, personal communication, October 4, 2019). This violation of trust had significant consequences and demonstrated how an OPM's lack of awareness could put the institution at risk.

\section{Organizational Change and Innovation}

The shift towards outsourcing online learning may be transformational for universities and colleges. Some refer to this shift as a disruptive innovation or organizational change to address a business's failure to compete in the marketplace (Bower \& Christensen, 1995). An integrated entrepreneurial culture or a work ethos that embraces change is embedded in organizational change (Clark, 1998; Deem, 2001).

University administrators in this study described the evolution of online learning that occurred in three phases. In the first phase, Big U offered for-credit distance learning through a syndicated national television. In the second phase, Big $U$ took a broader approach to deliver online education, including a failed attempt at a university-wide online education initiative, low-residency courses for study-abroad programs, and the acquisition of another college with a robust online learning arm. In the third phase, Big U experienced a period of innovation in online learning resulting from direct institutional support.

In this study, university administrators discussed several vital initiatives that marked the third wave from which OPM partnerships emerged. These markers are as follows (Participant 4, Participant 5, Participant 17, personal communications, October-November 2019):

- A period of governance that guided decision-making and policy development and prioritized and allocated resources towards online learning initiatives.

- An inventory of educational technology initiatives that led to the realization that a continuum of teaching and learning with technology existed at Big U, including online learning.

- An aggregated, university-wide discourse about online learning occurred through community events and educational technology demonstrations by faculty and administrators. 
- A policy that enabled students to take classes from any academic department in the university regardless of modality and the removal of a ban that previously prevented this scenario.

- A grant that provided financial resources to any school for various initiatives related to online learning, including staffing needs.

- University services designed to support academic departments pursuing online learning, especially those engaged with OPMs.

- The realization that the decision to engage with online learning was distributed and deference went to the deans of the various Schools at Big U.

The third phase was coordinated by academic and administrative leadership. The third phase resulted in a variety of funding, resources, and services that supported the outsourcing of online learning at Big U. For instance, the first school at Big U to establish an OPM partnership leveraged the grant program for human and financial capital.

In reflection of the third phase of online learning for the university, a vice president described this organizational change and innovation as follows:

But I think [by this university] demonstrating an investment in resources at the school level through the instructional designers, through the grant program, through things like the contract support, roles at the university that enable, that do not constrain growth, I think we have reached more people, we have viewed this as a learning program. (Participant 4, personal communication, October 18, 2019).

The shifting mission towards online learning at Big $U$ resulted from a prioritization of entrepreneurship and innovation. University administrators created infrastructure, established policies, and offered financial and human resources that aided the university in its shifting mission regarding online learning. The outsourcing of online learning was paramount and allowed the institution to move online quickly and at scale.

\section{RECCOMENDATIONS FOR INSTITUTIONS}

Outsourcing is not a nascent practice; in fact, outsourcing in education has roots dating back to the early 1900s (Abrams, 2016). However, the outsourcing of online learning through partnerships with OPMs is a new practice in higher education. Therefore, this in-depth case study is instructive for other higher education professionals because it demonstrates the mechanisms involved in these outsourcing relationships. Other university administrators and decision-makers can consider this case and its elements that are applicable to their institutions. Below are some recommendations for institutions considering an OPM partnership.

\section{Consider the Need for Outsourcing}

Is an OPM necessary to build and run an online program? Administrators in this study generally concluded that OPMs served a limited purpose. Some administrators saw the OPM partnership as a learning opportunity so that administrators could replicate the OPM's approach later. An OPM partnership may be a good fit for some institutions; however, OPM partnerships are not the only way to deliver online education. Therefore, it is essential for university decision-makers to consider this question earnestly and to explore alternative approaches to building online programs as part of the decision-making process. OPMs are opportunistic and, as part of their practice, present a simplified version of the services they provide. However, an OPM partnership is complex, and there is no abridged solution to delivering online education. Each institution is unique, and the decision to contract an OPM is contextual.

\section{Negotiate Informal Aspects of the Partnership}

The idea that the OPM was in full control of the partnership undergirded this study. Very few administrators in this study were privy to the OPM contracts. Nonetheless, the administrators described 
countless touchpoints with the OPMs that were informal and potential opportunities for negotiation practices. For example, administrators might ask to help hire OPM staff to ensure the proper credentialing if the professional profile is a concern. Therefore, it is essential to empower administrators to manage their work with OPM staff autonomously; in situations where administrators cannot negotiate the contracts, they will benefit from more control or influence over the OPM partnership's day-to-day activities.

\section{Establish a Centralized Alliance Management Function}

An alliance management function is a centralized group within an organization that is responsible for managing strategic partnerships. An alliance management function also contributes to the institutional "know-how" about strategic partnerships, helping to make the partnerships more successful (Kale \& Singh, 2007). At Big U, the OPM partnerships were managed at the individual school level, which contributed to some of the challenges administrators expressed about working with OPMs.

The university alliance management function helps staff coordinate and oversee the alliance (e.g., OPM partnerships; Kale \& Singh, 2007). Minimally, alliance management denotes a single point of contact for a partnership. Maximally, alliance management may represent a centralized unit designed to manage the complex nature of one or many strategic partnerships across an institution. Within higher education, alliance management functions will vary based on institutional structures. However, establishing a function with oversight for OPM partnerships promotes knowledge acquisition and knowledge sharing about the partnerships and creates a central resource to manage the partnerships' performance.

\section{Create a More Inclusive Decision-Making Process}

When evaluating OPMs, university decision-makers need to engage with those who will operationalize these partnerships: university administrators. A transparency of information and mission alignment for stakeholders involved in the partnership occurs when University administrators become part of the decisionmaking process (Abrams, 2016). The lack of clarity about contract terms was problematic for university administrators in this study. Administrators did not know how to evaluate the performance of the OPMs and had the minimal authority to address challenges in the partnership rightfully. University administrators did not understand the purpose of hiring an OPM, the OPM partnership's goals, or the terms by which to evaluate the contract. Closing the gap between decision making and decision implementation will improve mission alignment, improve communication, clarify roles and responsibilities, and reduce conflicts.

\section{Confirm Experiences Outside of Higher Education}

A common way to validate a potential service provider is through reference checks. Many administrators in this study shared that they consulted peer institutions and colleagues at Big U who had worked with OPMs. This collaboration represented the administrators' efforts to evaluate a potential OPM partner; however, their attempts only resulted in isomorphism by reinforcing the status quo. Thus, higher education professionals should consider cross-checking experiences in other industries that are more familiar with strategic partnerships, especially complex partnerships that involve the delivery of education in new ways.

\section{Implications for OPMs}

This study was designed to benefit universities; however, this study will undoubtedly help OPMs as well. To that end, the most critical implication for OPMs is to hire, build, and structure teams carefully so that teams better align with the postsecondary institution that OPM staff will support. An underlying tension exists between higher education and for-profit companies. This tension is heightened when academics are involved; thus, it is even more critical that OPMs understand this sensitivity and respond to it. OPM teams members must have the appropriate academic expertise (e.g., educational background and degree attainment), understand the academic discipline of the online program they will support, and understand how higher education works. University administrators evaluate OPM staff on these barometers of trust. When business supersedes academics, university administrators cannot identify with and lose trust in OPM staff. 


\section{SUMMARY}

This paper details the findings of a case study conducted at a 4-year private university in the United States. The study site was outsourcing online learning through university-industry partnerships with OPMs. The study findings provided information for other higher education administrators that are considering or currently engaging in university-industry partnerships.

\section{REFERENCES}

Abrams, S.E. (2016). Education and the commercial mindset. Harvard University Press. Retrieved from https://play.google.com/store/books/details?id=1I24CwAAQBAJ

Bower, J.L., \& Christensen, C.M. (1995). Disruptive technologies: Catching the wave. Harvard Business Review, 73(1), 45-53. https://doi.org/10.1016/0024-6301(95)91075-1

Clark, B.R. (1998). Creating entrepreneurial universities: Organizational pathways of transformation. Emerald.

Deem, R. (2001). Globalisation, new managerialism, academic capitalism and entrepreneurialism in universities: Is the local dimension still important? Comparative Education Review, 37(1), 7-20. https://doi.org/10.1080/03050060020020408

Entangled Solutions. (2020). Institutional change and impact map. Retrieved from https://www.entangled.solutions/coronavirus-he/

Giannini, S. (2020, February 6). Education is the bedrock of a just society in the post-COVID world. UNESCO. Retrieved from https://en.unesco.org/news/covid-19-education-bedrock-just-societypost-covid-world

Hill, P. (2019, September 27). Online program management: An updated view of the market landscape for 2019. Phil On Ed Tech. Retrieved from https://philonedtech.com/opm-updated-2019-marketlandscape/

Holon IQ. (2019, February 24). The anatomy of an OPM. Retrieved from https://www.holoniq.com/news/anatomy-of-an-opm/

Holon IQ. (2020, April 24). Global OPM and PPP market. Retrieved from https://www.holoniq.com/notes/the-7b-global-opm-and-academic-ppp-market/

Kale, P., \& Singh, H. (2007). Building firm capabilities through learning: The role of the alliance learning process in alliance capability and firm-level alliance success. Strategic Management Journal, 28(10), 981-1000. https://doi.org/10.1002/smj.616

Martin, M. (2000). Managing university-industry relations: A study of institutional practices from 12 different countries (Improving the managerial effectiveness of higher education institution series). International Institute for Educational Planning. https://unesdoc.unesco.org/ark:/48223/pf0000120290/PDF/120290eng.pdf.multi

Miles, M.B., \& Huberman, M.A. (1994). Qualitative Data Analysis: An Expanded Sourcebook. SAGE.

Miles, M.B., Huberman, M.A., \& Saldana, J. (2019). Qualitative data analysis (4th ed.). Sage.

Morgan, A.G. (2019, June 13). Market guide for online program management in higher education. Gartner.

Ren, H., Gray, B., \& Kim, K. (2009). Performance of international joint ventures: What factors really make a difference and how? Journal of Management, 35(3), 805-832. https://doi.org/10.1177/0149206308331165

Saldaña, J. (2016). The coding manual for qualitative researchers. Sage.

Seaman, J.E., Allen, E.I., \& Seaman, J. (2018). Grade increase: Tracking distance education in the united states. BABSON Survey Research Group. Retrieved from https://onlinelearningsurvey.com/reports/gradeincrease.pdf

Slaughter, S., \& Leslie, L.S.S. (1997). Academic capitalism: Politics, policies, and the entrepreneurial university. Johns Hopkins University Press. 